\title{
Reducing Psychotropic Use in Older Non-demented Spanish Care Home Residents: a Before-After Comparison of Three Methods
}

\author{
Curtis L. Petersen, $M P H^{7}$ (D), David Curto, MD, MBA ${ }^{2}$, Manish K. Mishra, MD, MPH', \\ Pedro Cano, $M D^{2}$, and William B. Weeks, $M D, P h D, M B A^{7}$ \\ ${ }^{1}$ The Dartmouth Institute for Health Policy and Clinical Practice, Lebanon, NH, USA; ${ }^{2}$ Sanitas Mayores (a subsidiary of Bupa, London, England), \\ Barcelona, Spain.
}

J Gen Intern Med 35(1):383-5

DOI: $10.1007 / \mathrm{s} 11606-019-05517-2$

(c) Society of General Internal Medicine 2019

\section{INTRODUCTION}

Recognizing that Spain has the highest rate of antipsychotic prescribing in Western Europe, ${ }^{1}$ the Spanish Geriatrics and Gerontology Society has encouraged reducing psychotropic medication use in long-term settings. ${ }^{2}$ In a quality improvement effort designed to do that, we found that administration of any of 3 interventions - application of STOPP/START criteria, medication-focused team rounds (TR), or a decision aid (DA) - effectively reduced psychotropic use among older Spanish nursing home residents with dementia, though STOPP/START criteria application was most effective. ${ }^{3}$

While care home residents with dementia are at risk for psychotropic overuse and their sequelae, so might be those without dementia. ${ }^{4}$ Therefore, we sought to analyze patients without dementia who obtained any of the 3 interventions we studied in Sanitas Mayores (Spain), a Bupa-affiliated aged care network.

\section{METHODS}

Subject and intervention selection and study implementation are detailed elsewhere. ${ }^{3}$ In brief, we examined the short-term effects of implementation of 3 aforementioned interventions on mean equivalent daily doses (MEDDs) of 3 psychotropic medication classes: antidepressants, anxiolytics, and antipsychotics. We limited our analysis to residents aged 70-99 who lacked an Electronic Health Record (EHR)-documented dementia diagnosis (no ICD-9 or ICD-10 dementia diagnosis; Short Portable Mental Status Questionnaire or Functioning Assessment Staging Scores lower than 5). Age, sex, length of time living in the care home, Charlson score, and the EHR-

This work has not been previously presented or published elsewhere.

Received April 26, 2019

Accepted October 22, 2019

Published online November 11, 2019 derived MEDD for each drug of interest were used to generate propensity scores; in each drug class, we matched 2 controls to each intervention resident. We used a mixed effects model to adjust for correlation within intervention sites and within patients over time. For each drug class/intervention combination, we compared MEDDs in the 4 weeks before intervention with those in the 4 weeks after intervention. During the same time frame, we examined 2 potential adverse effects: patient fall and physical restraint use rates We used R (v4.5), 2-tailed statistical significance of 0.05 , and report standardized differences in MEDD amounts and proportions. Dartmouth's IRB approved the study, which follows STROBE guidelines.

\section{RESULTS}

Intervention and matched control care home residents were similar at baseline, having similarly low prevalence of psychiatric disorders (Table 1). Relative to propensity-matched controls, all 3 interventions were associated with significant reductions in both antipsychotic MEDDs (STOPP/START, $-20.4 \%$ ( 15.6 milligram equivalents) [95\% confidence interval, $29.4 \%,-11.5 \%]$; DA, $-15.8 \%$ ( -49.6$)[-21.3 \%$, $10.5 \%]$; TR, $-36.5 \%(-160.0)[-51.3 \%,-21.7 \%])$ and anxiolytic MEDDs (STOPP/START, - 6.9\% (- 1.96) [$11.7 \%,-2.1 \%]$; DA, $-17.7 \%(-2.5)[-32.1 \%,-3.3 \%]$; TR, $-13.1 \%(-3.71)[-20.3 \%,-6.0 \%])$; none of the interventions was associated with significant reductions in antidepressant MEDDs (Fig. 1). Compared with controls, a higher proportion of intervention patients had antipsychotic (STOPP/ START, 24.1\%, $p<0.01$; TR, 53.1\%, $p<0.01$ ) and anxiolytic (STOPP/START, $13.0 \%, p<0.01$; TR, 23.0\%, $p<0.01$ ) medications discontinued. Post-intervention fall and physical restraint use did not differ (data not shown, all $p>0.1$ ).

\section{DISCUSSION}

Among older non-demented Spanish care home residents, the interventions we studied were followed by lower antipsychotic and anxiolytic dosing, but unchanged antidepressant dosing. In contrast to findings for care home residents with dementia, ${ }^{3}$ 
Table 1 Characteristics of Controls and Cases by Intervention and Medication. Controls Were Propensity Score Matched at a Rate of 2:1. We Found No Statistically Significant Differences between Controls and Cases Within a Particular Intervention/Medication Combination. SMD, Standardize Mean Difference

\begin{tabular}{|c|c|c|c|c|c|c|c|c|c|c|}
\hline \multirow{3}{*}{$\begin{array}{l}\text { Prescription } \\
\text { type }\end{array}$} & \multirow{3}{*}{$\begin{array}{l}\text { Baseline } \\
\text { characteristics }\end{array}$} & \multicolumn{9}{|c|}{ Intervention } \\
\hline & & \multicolumn{3}{|c|}{ Team rounds } & \multicolumn{3}{|c|}{ Decision aid } & \multicolumn{3}{|c|}{ STOPP/START } \\
\hline & & Controls & Cases & SMD & Controls & Cases & SMD & Controls & Cases & SMD \\
\hline \multirow{7}{*}{$\begin{array}{l}\text { Anti- } \\
\text { depressant }\end{array}$} & $N$ & 68 & 34 & & & 14 & & 144 & 72 & \\
\hline & $\begin{array}{l}\text { Age (years), mean } \\
\text { (SD) }\end{array}$ & $86.7(6.1)$ & $\begin{array}{l}86.8 \\
(6.3)\end{array}$ & 0.02 & $85.0(6.5)$ & $\begin{array}{l}86.4 \\
(6.3)\end{array}$ & 0.21 & $86.4(6.2)$ & $\begin{array}{l}86.9 \\
(5.7)\end{array}$ & 0.09 \\
\hline & Male, $n(\%)$ & $17(25.0)$ & $7(20.6)$ & 0.11 & $0(0)$ & $0(0)$ & NA & $29(20.1)$ & 13 (18.1) & 0.05 \\
\hline & $\begin{array}{l}\text { Years admitted, } \\
\text { mean (SD) }\end{array}$ & $3.9(3.8)$ & $3.6(2.6)$ & 0.08 & $2.1(2.0)$ & $2.2(1.7)$ & 0.02 & $3.1(3.1)$ & $3.4(3.0)$ & 0.11 \\
\hline & $\begin{array}{l}\text { Dose (mg/day), } \\
\text { mean (SD) }\end{array}$ & $11.6(9.9)$ & $11.7(8.6)$ & 0.01 & $\begin{array}{l}18.7 \\
(13.0)\end{array}$ & $\begin{array}{l}18.6 \\
(14.3)\end{array}$ & 0.01 & $12.4(9.1)$ & $\begin{array}{l}13.4 \\
(8.5)\end{array}$ & 0.12 \\
\hline & $\begin{array}{l}\text { Charlson, mean } \\
\text { (SD) }\end{array}$ & $\begin{array}{l}0.58 \\
(0.92)\end{array}$ & $\begin{array}{l}0.76 \\
(0.74)\end{array}$ & 0.22 & $\begin{array}{l}0.71 \\
(0.94)\end{array}$ & $\begin{array}{l}0.79 \\
(1.12)\end{array}$ & 0.07 & $\begin{array}{l}0.68 \\
(0.86)\end{array}$ & $\begin{array}{l}0.67 \\
(0.80)\end{array}$ & 0.01 \\
\hline & $\begin{array}{l}\text { Psychiatric } \\
\text { diagnoses, } n(\%)\end{array}$ & $13(19.1)$ & $6(17.6)$ & 0.04 & $10(35.7)$ & $3(21.4)$ & 0.32 & $25(17.4)$ & $10(13.9)$ & 0.10 \\
\hline \multirow[t]{7}{*}{ Anxiolytic } & $N$ & 96 & 48 & & 42 & 21 & & 192 & 96 & \\
\hline & $\begin{array}{l}\text { Age (years), mean } \\
\text { (SD) }\end{array}$ & $87.1(6.1)$ & $\begin{array}{l}86.95 \\
(5.9)\end{array}$ & 0.03 & $84.8(7.1)$ & $\begin{array}{l}85.4 \\
(6.6)\end{array}$ & 0.09 & $86.9(5.8)$ & $\begin{array}{l}86.2 \\
(6.6)\end{array}$ & 0.12 \\
\hline & Male, $n(\%)$ & $13(13.5)$ & $6(12.5)$ & 0.03 & $10(23.8)$ & $3(14.3)$ & 0.24 & $37(19.3)$ & $21(21.9)$ & 0.06 \\
\hline & $\begin{array}{l}\text { Years admitted, } \\
\text { mean (SD) }\end{array}$ & $4.0(3.6)$ & $4.1(3.2)$ & 0.03 & $2.7(2.8)$ & $2.7(2.9)$ & 0.02 & $2.8(3.1)$ & $2.8(2.5)$ & 0.01 \\
\hline & $\begin{array}{l}\text { Dose (mg/day), } \\
\text { mean (SD) }\end{array}$ & $\begin{array}{l}18.6 \\
(10.6)\end{array}$ & $\begin{array}{l}18.2 \\
(11.1)\end{array}$ & 0.03 & $12.5(9.2)$ & $\begin{array}{l}12.8 \\
(8.1)\end{array}$ & 0.03 & $15.0(9.5)$ & $\begin{array}{l}15.0 \\
(10.8)\end{array}$ & $\stackrel{<}{0.01}$ \\
\hline & $\begin{array}{l}\text { Charlson, mean } \\
\text { (SD) }\end{array}$ & $\begin{array}{l}0.71 \\
(0.81)\end{array}$ & $\begin{array}{l}0.75 \\
(0.81)\end{array}$ & 0.05 & $\begin{array}{l}0.61 \\
(0.78)\end{array}$ & $\begin{array}{l}0.52 \\
(0.68)\end{array}$ & 0.11 & $\begin{array}{l}0.58 \\
(0.75)\end{array}$ & $\begin{array}{l}0.55 \\
(0.74)\end{array}$ & 0.04 \\
\hline & $\begin{array}{l}\text { Psychiatric } \\
\text { diagnoses, } n(\%)\end{array}$ & $19(19.8)$ & $16(33.3)$ & 0.31 & $6(14.3)$ & $6(28.6)$ & 0.35 & 34 (17.7) & 15 (15.6) & 0.06 \\
\hline \multirow[t]{6}{*}{ Anti-psychotic } & $\begin{array}{l}N \\
\text { Age (years), mean } \\
\text { (SD) }\end{array}$ & $\begin{array}{l}32 \\
86.3(7.2)\end{array}$ & $\begin{array}{l}16 \\
86.8 \\
(6.6)\end{array}$ & 0.08 & $\begin{array}{l}16 \\
82.0(7.9)\end{array}$ & $\begin{array}{l}8 \\
81.3 \\
(8.9)\end{array}$ & 0.07 & $\begin{array}{l}54 \\
85.1(7.9)\end{array}$ & $\begin{array}{l}27 \\
85.7 \\
(6.3)\end{array}$ & 0.10 \\
\hline & Male, $n(\%)$ & $5(15.6)$ & $2(12.5)$ & 0.09 & $10(62.5)$ & $5(62.5)$ & $<$ & $19(35.2)$ & $10(37.0)$ & 0.04 \\
\hline & $\begin{array}{l}\text { Years admitted, } \\
\text { mean (SD) }\end{array}$ & $3.8(3.9)$ & $3.5(3.3)$ & 0.07 & $3.0(2.1)$ & $3.3(4.1)$ & 0.12 & $2.9(3.4)$ & $3.0(3.4)$ & 0.03 \\
\hline & $\begin{array}{l}\text { Dose (mg/day), } \\
\text { mean (SD) }\end{array}$ & $\begin{array}{l}244.6 \\
(260.0)\end{array}$ & $\begin{array}{l}309.6 \\
(337.5)\end{array}$ & 0.22 & $\begin{array}{l}285.8 \\
(291.6)\end{array}$ & $\begin{array}{l}271.1 \\
(253.1)\end{array}$ & 0.05 & $\begin{array}{l}274.0 \\
(310.2)\end{array}$ & $\begin{array}{l}231.2 \\
(218.7)\end{array}$ & 0.16 \\
\hline & $\begin{array}{l}\text { Charlson, mean } \\
\text { (SD) }\end{array}$ & $\begin{array}{l}0.69 \\
(0.64)\end{array}$ & $\begin{array}{l}0.88 \\
(0.96)\end{array}$ & 0.23 & $\begin{array}{l}0.12 \\
(0.34)\end{array}$ & $\begin{array}{l}0.38 \\
(0.74)\end{array}$ & 0.43 & $\begin{array}{l}0.52 \\
(0.64)\end{array}$ & $\begin{array}{l}0.56 \\
(0.70)\end{array}$ & 0.06 \\
\hline & $\begin{array}{l}\text { Psychiatric } \\
\text { diagnoses, } n(\%)\end{array}$ & $11(34.4)$ & $5(31.2)$ & 0.07 & $6(37.5)$ & $3(37.5)$ & $\begin{array}{l}< \\
0.01\end{array}$ & $13(24.1)$ & $6(22.2)$ & 0.04 \\
\hline
\end{tabular}

for those without dementia, application of STOPP/START criteria was generally less effective than the other interventions.

These findings make sense: patients without cognitive impairment might respond better to interventions requiring active engagement (like TRs or DAs); those cognitively impaired might benefit from objectively applied medication reviews requiring less active engagement, like STOPP/START criteria. Further, antipsychotic and anxiolytic medications might have been intended for short-term use, but had not been reviewed.

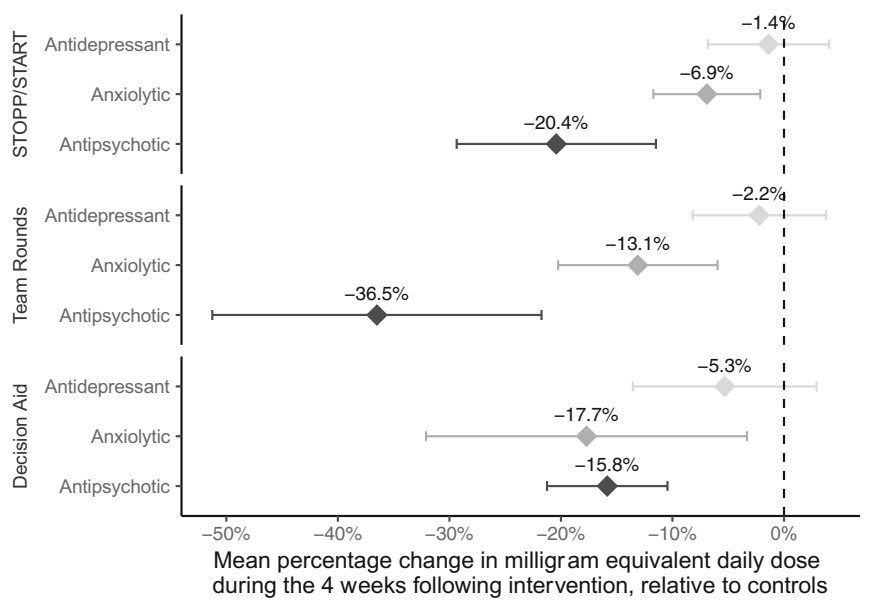

Figure 1 Average adjusted effect of each intervention on percentage change in daily equivalent doses of measured medications. 
This analysis has the same limitations as the parent study, which used EHR data from a single organization, was observational, allowed clinicians to decide whether to use an intervention, did not examine clinician attitudes, and examined the impact of only 3 tools on only 3 drug classes for a relatively short time period.

Nonetheless, our findings suggest there are opportunities to regularly re-evaluate psychotropic medication use and dosing among Spanish care home residents who do not have dementia, just as there are among those who do. Considering elders' independence from overuse of medications as a human rights issue $^{5}$ and their higher mortality risk when using antipsychotics, ${ }^{2}$ Spanish authorities might track psychotropic medication among all care home residents - regardless of whether they have dementia - and encourage regular review of psychotropic use and dosing.

Corresponding Author: Curtis L. Petersen, MPH; The Dartmouth Institute for Health Policy and Clinical Practice, 5th Floor Williamson Translational Research Building, Dartmouth Hitchcock Medical Center, 1 Medical Center Dr, Lebanon, NH 03766, USA (e-mail: Curtis.L. Petersen.GR@dartmouth.edu).

Author Contributions The listed authors contributed significantly to the conceptualization, design, data collection, analysis, interpretation and manuscript development.

Funding Information Drs. Weeks, Mishra, and Mr. Petersen received funding to conduct the work described in this manuscript. Drs. Curto and Cano are employees of Sanitas. Mr. Petersen is also funded by the Burroughs Wellcome Fund Big Data in the Life Sciences Program at Dartmouth.

\section{Compliance with Ethical Standards:}

Conflict of Interest: Sanitas Mayores, a subsidiary of Bupa, provided funding to Drs. Weeks, Mishra, and Mr. Petersen for the study. Drs. Curto and Cano are employees of Sanitas.

\section{REFERENCES}

1. Janus SIM, van Manen JG, Ijzerman MJ, Zuidema SU. Psychotropic drug prescriptions in Western European nursing homes. Int Psychogeriatr. 2016;28(11):1775-90.

2. Ramos Cordero P, Lopez Trigo JA, Maillo Pedraz H, Paz Rubio JM. [Physical and pharmacological restraints in geriatric and gerontology services and centers]. Revista espanola de geriatria y gerontologia. 2015;50(1):35-8. Epub 2014/12/03. doi: https://doi.org/10.1016/j.regg. 2014.09.006

3. Weeks WB, Mishra MK, Curto D, Petersen CL, Cano P, Hswen Y, Serra SV, Elwyn G, Godfrey MM, Soro PS, Tomás JF. Comparing three methods for reducing psychotropic use in older demented spanish care home residents. J Am Geriatr Soc 2019;67:1444-53. https://doi.org/10.1111/ jgs. 15855.

4. Wills P, Claesson CB, Fratiglioni L, Fastbom J, Thorslund M, Winblad B. Drug use by demented and non-demented elderly people. Age and ageing. 1997;26(5):383-91. Epub 1997/11/14.

5. "They want docile." How nursing homes in the United States overmedicate people with dementia. https://www.hrw.org/report/2018/02/05/theywant-docile/how-nursing-homes-united-states-overmedicate-people-dementia. United States of America: Human Rights Watch, 2018.

Publisher's Note: Springer Nature remains neutral with regard to jurisdictional claims in published maps and institutional affiliations. 